\title{
Konzeption einer Intranetstrategie im Rahmen des internen Marketings und Knowledge Managements
}

\author{
Stefan Kaht
}

\section{Geleitwort}

Die nachfolgend beschriebene, herausragende Diplomarbeit des Studenten der Wirtschaftsinformatik an der TFH Wildau, Stefan Kaht, hatte das Ziel, für ein international tätiges Beratungsunternehmen konzeptionelle Vorstellungen für eine Intranetstrategie zu erarbeiten. Dabei verfolgte der Kandidat ein vierstufiges Konzept für seine Untersuchungen. Nach einer Ist-Analyse der vorhandenen organisatorischen Regelungen und technischen Gegebenheiten wurden vorhandene Schwachstellen lokalisiert. Danach erfolgte auf Basis einer Befragung von Content-Providern eine Anforderungsanalyse, um daraus in einem dritten Schritt vor allem jene organisatorischen Prozesse zu bestimmen, die als grundlegend für die inhaltliche Konzeption der Intranetstrategie anzusehen sind.

Abschließend skizzierte der Kandidat in prototypischer Form die vorgesehene technische Konzeption, die bei der Umsetzung der Untersuchungsergebnisse und -empfehlungen verfolgt werden sollte.

Die Diplomarbeit überzeugt vor allem durch die Komplexität und Sorgfalt der Untersuchungen, mit der die verschiedenen Lösungsvorschläge erarbeitet und begründet werden. Sie ist eine gelungene Synthese aus der Anwendung modernen Wissens für eine hochaktuelle, in vielen Details noch keineswegs abschließend gelöste Fragestellung der Unternehmenspraxis.

Der Kandidat musste dabei nicht nur nachweisen, dass er sich auf Spezialgebieten moderner Wirtschaftsinformatik auskennt. Bemerkenswert ist vor allem, dass in den Analysen und Lösungsvorschlägen zusätzliches Wissen auf den Gebieten Change Management, Knowledge Management und Human Ressource Management schöpferisch und fruchtbar mit der Intranetproblematik verbunden wurden.

\section{Einführung}

Es ist heutzutage durchaus üblich, die Wirtschaftswelt als Dienstleistungsgesellschaft oder als Informationsgesellschaft zu bezeichnen - unstrittig ist, dass der wesentliche Rohstoff für die ökonomische Entwicklung heute in den Köpfen der Menschen liegt.

Diese Diplomarbeit wurde in einem weltweit führenden Dienstleistungsunternehmen erstellt. Im Rahmen einer globalen Strategie ist ein Wissensmanagementsystem namens KWorld im Einsatz, das es Mitarbeitern ermöglicht, auf eine zentrale Ressource von unternehmenswichtigen Informationen zuzugreifen.

Der Zentralbereich Informationstechnologie (ZI), als Teil des National Office, ist für die deutschlandweiten strategischen Entscheidungen und Entwicklungen im EDVBereich verantwortlich. Es handelt sich gewissermaßen um einen Dienstleister in einem Dienstleister, dessen Kunden die Mitarbeiter aller deutschen Unternehmensniederlassungen sind.

Für die Präsentation von Zl-Informationen, z. B. über Abteilungen, Anwendungen und den gesamten Nutzersupport, aber auch für die interne Kommunikation betreibt ZI ein eigenes Intranet, das IT-Web. Bei Einführung dieses Systems wurden vor allem technische Parameter berücksichtigt und konzeptionell keine klaren Rahmenbedingungen bzw. Anforderungen formuliert. Die Intranetlösung weist eine Menge Schwächen auf, welche bei der täglichen Arbeit das Einstellen von Informationen sehr schwierig gestalten. Dieser Missstand war Anlass für die vorliegende Diplomarbeit. Aufbauend auf einer Dokumentation der momentanen Unzulänglichkeiten zeigt die Diplomarbeit einen Neuansatz fur die Nutzung des Intranets im Rahmen des Internen Marketings und Wissensmanagements. Ebenso wird die technische Umsetzung dieser Konzeption dargestellt.

\section{Vorbetrachtungen zur Konzeption}

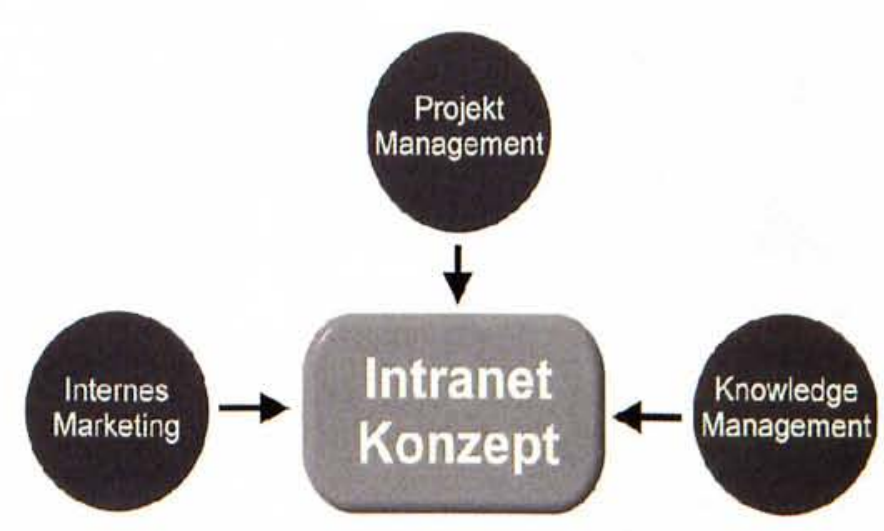

In Bezug auf das Thema konnten drei externe Einflussfaktoren identifiziert werden, die bei der Erstellung des Intranetkonzepts und somit der Diplomarbeit eine wesentliche Rolle spielten. Aus diesen, in der Grafik ersichtlichen Einflussfaktoren, ließ sich ein geeigneter Aufbau des Intranetkonzepts ableiten, der auch einen direkten Einfluss auf die chronologische Durchfuihrung der Diplomarbeit hatte. 


\section{Aufbau eines Intranetkonzepts}

Im Rahmen des Projekt-Managements wird ein Intranetkonzept aus drei Komponenten aufgebaut: Organisation, Inhalt und Technik. Die durchgefuihrten theoretischen Betrachtungen für Internes Marketing und Knowledge Management fließen dabei in Betrachtungen der einzelnen Komponenten ein. Die Grafik zeigt die Bedeutung der Komponenten, ihre zeitliche Abfolge bei der Erstellung und die Integration der Einflussfaktoren eines Intranetkonzepts. Basierend auf diesen gewonnen Erkenntnissen konnte eine Vorgehensweise definiert werden.

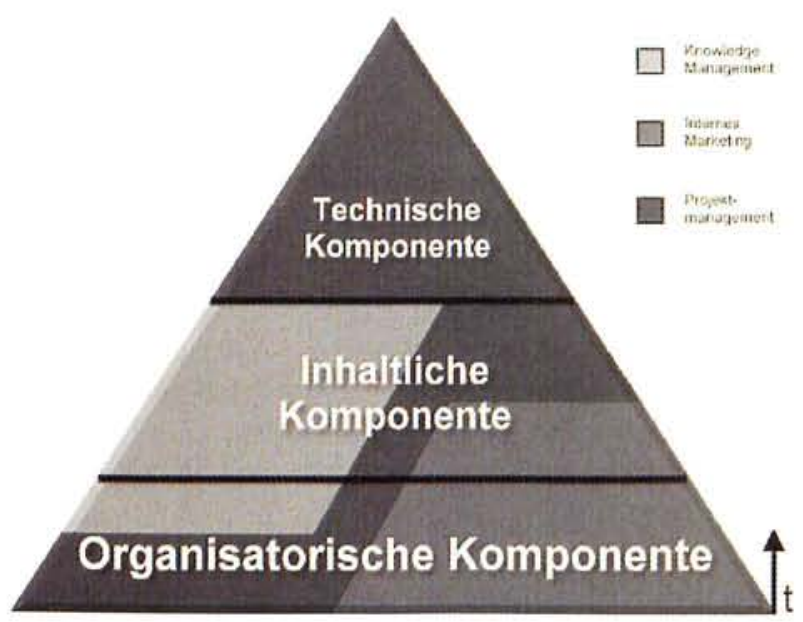

\section{Vorgehensweise}

Es wurde als sinnvoll erachtet, das Vorgehen aus dem Prototyping-Modell fuir Softwareentwicklungen abzuleiten und dieses der Problemstellung anzupassen. Dabei wird für die eigentliche Erstellung des Intranetkonzepts kein, wie beim Prototyping uiblich, iterativer Prozess genutzt, sondern ein linearer Verlauf, der nach der Umsetzung eines Prototypen endet. Den zeitlichen Ablauf der Teilprozesse soll die folgende Grafik verdeutlichen.

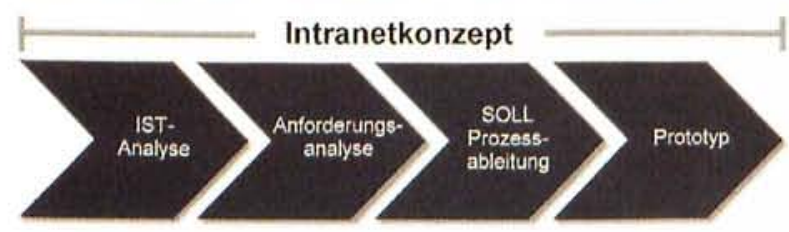

Es wird vorausgesetzt, dass die Themeninitiierung bereits stattgefunden hat, die den Anlass für die Diplomarbeit gab und die Rahmenbedingungen für die Durchführung festgelegt hat.

Jeder Teilprozess wurde dabei, in Anlehnung an das ermittelte Modell „Aufbau eines Intranetkonzepts“, auf organisatorische Prozesse und Regelungen, inhaltliche sowie technische Betrachtungen untersucht.

\section{Ist-Analyse}

Im ersten Schritt wurden im Rahmen der Ist-Analyse die aktuelle Situation im ZI untersucht und die Schwachstellen des derzeit im Einsatz befindlichen Systems fixiert.
Detailliert wurde festgehalten, wie die aktuelle Verteilung der Verantwortlichkeiten auf organisatorischer Ebene aufgegliedert ist. In den Fachabteilungen konnten 4 Teilprozesse identifiziert werden: Erstellung, Qualitätssicherung, Freigabe und Struktureinordnung.

Aus inhaltlicher Sicht konnte ein Übermaß an veralteten Informationen festgestellt werden.

Die technische Betrachtung ergab eine unkoordinierte Nutzung der vorhandenen technischen Ressourcen und Infrastruktur. Das eingesetzte System, basierend auf dem Hyperwave Information Server, war fehlerhaft und die Integration in andere existierende Systeme war so gut wie unmöglich. Das größte festgestellte Manko des existierenden Systems war seine überdurchschnittlich schlechte Performance.

\section{Anforderungsanalyse}

Im zweiten Schritt wurden die Nutzeranforderungen im Rahmen der Anforderungsanalyse aufgenommen. Besondere Aufmerksamkeit erhielten die Anforderungen der Content Provider, da sie das entscheidende Kriterium jeder Intranetstrategie sichern: die Qualität des Inhalts! Zu diesem Zweck wurden mit einer repräsentativen Gruppe der Content Provider Interviews durchgefuihrt, welche als Ergebnis empirisch auswertbare Protokolle lieferten, die sich ideal für die Soll-Definition eigneten.

Organisatorisch sollten vor allem kompetente Ansprechpartner forciert werden, um das System nutzbar zu machen und den Mitarbeitern eine transparente Vorgehensweise anzubieten.

Die inhaltliche Hauptanforderung war die logische und intuitive Strukturierung der im Intranet abgelegten Informationen. Es muss eine Struktur existieren, die den Anwender bei der Suche nach Informationen unterstützt und eine einfache Einordnung beim Einstellen von Dokumenten ermöglicht. Dabei muss die Struktur im Detail flexibel sein, um individuelle Wünsche der Content Provider zu realisieren und für zukünftige Anforderungen offen sein.

Bei den technischen Anforderungen ging es vor allem um Funktionalitäten des Intranet-Systems zur Unterstuitzung der Content Provider und Content Customer.

\section{Sollprozessableitung}

In der Sollprozessableitung, als drittem Schritt, konnten aus den Erkenntnissen der Ist-Analyse und der Anforderungsanalyse vor allem organisatorische Prozesse definiert werden, welche die Grundlage einer Intranetstrategie bilden.

Auf organisatorischer Ebene wurden, soweit die Ressourcen verfuigbar waren, Verantwortungen verteilt und ein allgemeines Vorgehen definiert.

Für die inhaltliche Verwaltung konnten Empfehlungen zur qualitativen Verbesserung erarbeitet werden. Im Wesentlichen wurden Vorschläge fuir zukünftige Inhalte und die Strukturierung dieser formuliert.

Bei der technischen Betrachtung galt es zu prïfen, ob sich die Anforderungen in KWorld abbilden lassen, was 
die Administration eines eigenen Intranets überfliissig machen wiirde. Die Auswertung ergab, die derzeitige Softwarelösung und somit ein eigenes ZI-internes Intranet weiter im Einsatz zu lassen unter der Bedingung, dessen Schwächen kontinuierlich zu beseitigen und die Serverinfrastruktur zu verbessern. Entscheidend für diese Erkenntnis war die Feststellung, dass ZI keine Informationsplattform, sondern eine Supportplattform benötigt.

\section{Prototyp}

Zur Wahrung der Corporate Identity und somit der Integration des ZI-internen Intranets in das KWorld System wurde im vierten und letzten Schritt ein Prototyp implementiert, der von $\mathrm{ZI}$ angebotene Informationen unter der KWorld-Oberfläche darstellt.

Die Erstellung eines Prototypen soll einen ersten praktischen und visuellen Eindruck von den schriftlichen Betrachtungen vermitteln, die während der Diplomarbeit durchgeführt wurden.

\section{Resümee und Ausblick}

Vor allem im Rahmen der Globalisierung gewinnt die Steuerung der internen Informations- und Kommunikationsflüsse immer mehr an Bedeutung.

Die Weiterentwicklung des Intranets endet allerdings nicht mit dem erstellten Konzept. Dieses bildet lediglich die Grundlage für die zukünftige Entwicklung des Internen Marketings und Wissensmanagements innerhalb des ZI. Im Anschluss an die Diplomarbeit muissen in einem regelmäßigen Turnus die Anforderungen der Interessengruppen aufgenommen und mit der aktuellen IstSituation abgeglichen werden. Zu diesem Zweck kann die für diese Diplomarbeit verwendete Vorgehensweise als iterativer Prozess genutzt werden.

Zukünftig sollte vor allem der Einsatz eines einzigen Systems furr alle Mitarbeiter des Unternehmens anvisiert werden. Ein eigenes Intranet bietet sich nur solange an, wie KWorld die Anforderungen des ZI nicht abbilden kann. Die aktuelle Entwicklung zeigt, dass KWorld ständig weiterentwickelt wird und die Freigabe der Version 2.0 kurz bevorsteht. Es ist mit einer Vielzahl an Verbesserungen zu rechnen und vielleicht kann schon in der Version 3.0 auf das Zl-interne Intranet verzichtet werden.

Weiterhin müssen die einzelnen Niederlassungen und die dort vorhandenen Informatikabteilungen stärker in das ZI-interne Intranet involviert werden, um die deutschlandweiten Bedürfnisse zu erfassen und in eine Gesamtstrategie aufzunehmen.

\section{Autor}

\section{Stefan Kaht}

Technische Fachhochschule Wildau Studiengang Wirtschaftsinformatik Tel. (0 30) 63409315 